\title{
Are older people more vulnerable to long-term impacts of disasters?
}

This article was published in the following Dove Press journal:

Clinical Interventions in Aging

7 December 2016

Number of times this article has been viewed

\author{
Hassan Rafiey' \\ Yadollah Abolfathi \\ Momtaz ${ }^{2,3}$ \\ Fardin Alipour ${ }^{1}$ \\ Hamidreza Khankeh ${ }^{4}$ \\ Shokoufeh Ahmadi ${ }^{4}$ \\ Mohammad Sabzi \\ Khoshnami' \\ Sharifah Azizah Haron ${ }^{3}$ \\ 'Research Center of Social Welfare \\ Management, Department of Social \\ Work, 'Iranian Research Center on \\ Aging, University of Social Welfare \\ and Rehabilitation Sciences, Tehran, \\ Iran; ${ }^{3}$ Malaysian Research Institute on \\ Ageing (MyAgeing), Universiti Putra \\ Malaysia, Serdang, Selangor, Malaysia; \\ ${ }^{4}$ Department of Health in Emergency \\ and Disaster, University of Social \\ Welfare and Rehabilitation Sciences, \\ Tehran, Iran
}

Background: Despite the growing interest in the study of disasters, there is limited research addressing the elderly population that lead to prejudiced beliefs that older adults are more vulnerable to disasters than younger adults. This study aimed to compare positive mental health between elderly and young earthquake survivors.

Method: Data for this study, consisting of 324 earthquake survivors, were obtained from a population-based cross-sectional survey conducted in Iran, 2015. The long-term effect of earthquake was assessed using the Mental Health Continuum-Short Form questionnaire. A one-way multivariate analysis of covariance (MANCOVA) using SPSS (version 22) was used in data analysis.

Results: Older adults scored significantly a higher level of overall positive mental health (mean $[\mathrm{M}]=34.31$, standard deviation $[\mathrm{SD}]=10.52)$ than younger age group $(\mathrm{M}=27.48, \mathrm{SD}=10.56$, $t=-4.41 ; P<0.001)$. Results of MANCOVA revealed a statistically significant difference between older and young adults on the combined positive mental health subscales $\left(F_{(3,317)}=6.95\right.$; $P<0.001$ ), after controlling for marital status, sex, and employment status.

Conclusion: The present findings showing a higher level of positive mental health among elderly earthquake survivors compared with their younger counterparts in the wake of natural disasters suggest that advancing age per se does not contribute to increasing vulnerability.

Keywords: aged, earthquakes, mental health, post-disaster, resiliency, vulnerability

\section{Introduction}

Natural disasters, as the greatest challenges for human societies, affect millions of people every year around the world. ${ }^{1}$ In this case, older adults are among the most vulnerable groups to the immediate impact of natural disasters. Several studies have found that physical well-being of older adults is more affected by natural disasters compared with their younger counterparts. ${ }^{2-4}$ Although the short-term effect of the disasters on the physical well-being of older adults has been largely studied and proven that they are more vulnerable because of decreased sensory awareness, physical impairment, chronic medical conditions, and socioeconomic limitations experienced by many of the elderly, ${ }^{5-7}$ conclusions about the long-term psychological effects of natural disasters on older adults have been inconclusive. Some previous studies reported that the elderly are more likely vulnerable to psychological problems. ${ }^{4,8,9}$ However, other studies found that the elderly are less vulnerable to psychological impacts of disasters compared to younger survivors. ${ }^{10-12}$

There are two perspectives to explain older adults' reactive to stressful events, including disasters. First perspective includes the maturation theory and the inoculation theory. The maturation theory proposes that older adults are less emotionally reactive to post-disaster distress. According to the maturation theory, older adults have more
Correspondence: Yadollah Abolfathi Momtaz Univer on Aging, iversity of Social Welfare and Daneshjo Blvd, Evin 19857I3834, Tehran, Iran

Email yabolfathi@gmail.com

Sharifah Azizah Haron Malaysian Research Institute on Ageing (MyAgeing) Universiti Putra Malaysia, Serdang, Selangor 43400 UPM, Malaysia Email sh.azizah@upm.edu.my (c) (1) (2) 2016 Rafiey et al. This work is published and licensed by Dove Medical Press Limited. The full terms of this license are available at https://www.dovepress.com/terms.php
and incorporate the Creative Commons Attribution - Non Commercial (unported, v3.0) License (http://creativecommons.org/licenses/by-nc/3.0/). By accessing the work you $\mathrm{BY}$
hC and incorporate the Creative Commons Attribution - Non Commercial (unported, v3.0) License (http://creativecommons.org/licenses/by-nc/3.0/). By accessing the work you for commercial use of this work, please see paragraphs 4.2 and 5 of our Terms (https://www.dovepress.com/terms.php). 
mature coping styles, which protect them against stressors. Therefore, elderly people are less reactive to stressful life events. Additionally, the inoculation theory suggests that previous experience with disaster provides an inoculation against strong emotional reaction to similar disasters. The second perspective that claims older adults are more vulnerable to natural disasters than younger people includes the resource theory and the exposure theory. According to the resource theory, elderly people are not easily able to recover because of lower socioeconomic status and weak functional capacity. Similarly, the exposure theory argues that older adults are not easily aware of the disaster signal; therefore, they are more likely to experience a greater sense of deprivation resulting from their losses. ${ }^{13-16}$

The present study was conducted to compare the longterm psychological impact of disaster between elderly and young earthquake survivors. In this study, positive mental health was considered as an index of the long-term psychological effect of earthquake. Positive mental health includes the presence of positive feelings (emotional well-being), positive functioning in individual life (psychological wellbeing), and community life (social well-being). ${ }^{17}$ This is the first study that aimed to examine the post-disaster mental health differences between elderly and younger adult survivors $\sim 3$ years after the 2012 East Azerbaijan earthquakes in Iran.

\section{Methodology}

Data for this study were obtained from a population-based cross-sectional survey conducted in 2015 on the 2012 East Azerbaijan earthquakes. On August 11, 2012, at 16:53 and 17:04 local time, two earthquakes measuring magnitudes of 6.4 and 6.3 on the Richter scale hit Ahar, Heris, and Varzaqan in Eastern Azerbaijan province, which led to 303 deaths and 2,600 injuries, and affected 60,000 people across 182 villages. ${ }^{18}$

The study was conducted in August 2015 (3 years after the earthquake) using a self-administered questionnaire. Participants were eligible to participate if they had been directly exposed to the earthquake in 2012. The study sample consisted of 324 earthquake survivors (56 older adults and 268 younger adults). A two-stage proportional cluster random sampling technique was used to obtain a representative sample of earthquake-stricken areas. Data were collected using a face-to-face interview by trained enumerators in respondents' homes, with a response rate of $90 \%$. Only one resident aged $\geq 18$ years from each household was randomly chosen and interviewed.

\section{Measurements}

The assessment questionnaire consisted of two parts. The first part included information about basic demography and socioeconomic status. The second part was the Mental Health Continuum-Short Form (MHC-SF) questionnaire, a well-validated and widely used measure of positive mental health. ${ }^{19-21}$ The MHC-SF consists of 14 items and measures emotional well-being (defined as life satisfaction), social well-being (social acceptance, social contribution, social actualization, social integration, and social coherence), and psychological well-being (autonomy, environmental mastery, personal growth, positive relations with others, purpose in life, and self-acceptance). The scale has demonstrated good validity and reliability across several countries including Iran. ${ }^{19,22}$

In this study, the MHC-SF showed good reliability: Cronbach's alpha coefficient were 0.91 for total scale and its three subscales (emotional well-being [0.86], social wellbeing [0.85], and psychological well-being [0.88]).

\section{Ethical considerations}

The study was conducted in accordance with the Helsinki Declaration, World Medical Association and approved by the Medical Research Ethics of University of Social Welfare and Rehabilitation Science Tehran, Iran. Informed consent was obtained by explaining the aim and process of the study, orally or in written. Information was kept confidential and participants had the right to withdraw at each stage of the research.

\section{Statistical analysis}

Data were analyzed using the statistical software package SPSS, version 22. Study variables were presented as either the mean $(\mathrm{M})$ or frequency. A two-tailed $P \leq 0.05$ was considered statistically significant. In order to reduce the risk of inflation from performing separate analyses of variance, a one-way multivariate analysis of covariance (MANCOVA) was used to examine differences in positive mental health (emotional, social, and psychological) scores between older adult survivors and young survivors, after controlling for marital status, sex, and employment status.

\section{Results}

The elderly group comprised 56 community-dwelling elderly persons aged $\geq 60$ years. The young group (aged $18-30$ years) included 268 respondents with a mean age of 25.32 (standard deviation $[\mathrm{SD}]=3.41$ ) years. Table 1 displays the sociodemographic characteristics and positive mental health status of the subjects by age group. 
Table I Descriptive characteristics of the subjects by age group

\begin{tabular}{|c|c|c|c|c|}
\hline \multirow[t]{2}{*}{ Variables } & \multicolumn{2}{|c|}{ Young group } & \multicolumn{2}{|c|}{ Older group } \\
\hline & n (\%) & $M(S D)$ & n (\%) & $M(S D)$ \\
\hline Age, years & & $25.32(3.4 I)$ & & $68.36(6.68)$ \\
\hline \multicolumn{5}{|l|}{ Sex } \\
\hline Female & $102(38.1)$ & & $24(42.9)$ & \\
\hline Male & $166(61.9)$ & & $32(57.1)$ & \\
\hline \multicolumn{5}{|l|}{ Marital status } \\
\hline Unmarried & 119 (44.4) & & $25(44.6)$ & \\
\hline Married & $149(55.6)$ & & $31(55.4)$ & \\
\hline \multicolumn{5}{|c|}{ Employment status } \\
\hline Government & $158(59.0)$ & & $27(48.2)$ & \\
\hline Farming & II $0(41.0)$ & & $29(51.8)$ & \\
\hline \multicolumn{5}{|c|}{ Positive mental health } \\
\hline $\begin{array}{l}\text { Emotional } \\
\text { well-being }\end{array}$ & & $6.00(2.87)$ & & $7.32(2.98)$ \\
\hline Social well-being & & $9.66(4.48)$ & & II.75 (4.37) \\
\hline $\begin{array}{l}\text { Psychological } \\
\text { well-being }\end{array}$ & & I I.82 (5.49) & & I5.24 (4.74) \\
\hline Total & & $27.48(10.56)$ & & 34.31 ( 10.52$)$ \\
\hline
\end{tabular}

Abbreviations: M, mean; SD, standard deviation.

Elderly people scored significantly higher levels of positive mental health $(\mathrm{M}=34.31, \mathrm{SD}=10.52)$ than younger participants $(\mathrm{M}=27.48, \mathrm{SD}=10.56 ; t=-4.41 ; P<0.001)$.

Results of data screening showed no univariate or multivariate outliers. The findings of the assessment of normality, linearity, and multicollinearity were satisfactory. In addition, a series of Pearson correlations were conducted between all of the dependent variables to examine the assumption that the dependent variables would be moderately correlated with each other. As shown in Table 2, a meaningful pattern of correlations was observed among all of the dependent variables, suggesting the appropriateness of a MANCOVA. Additionally, Box's test of equality of covariance was not significant (Box's $\mathrm{M}=9.20 ; P=0.173)$. This means that the assumption of homogeneity of variance-covariance matrices has been met, which tests the null hypothesis that the covariance matrices between the groups are equal. Although Box's M was not significant, the Pilli's criterion was reported because it is more robust and appropriate when there are unequal sample sizes.

As presented in Table 3, the results of MANCOVA revealed a statistically significant difference between older

Table 2 Means, SDs, and correlation matrix of the well-being subscales

\begin{tabular}{llllll}
\hline Dependent variables & $\mathbf{I}$ & $\mathbf{2}$ & $\mathbf{3}$ & $\mathbf{M}$ & SD \\
\hline Emotional & & $0.48^{* *}$ & $0.60^{* *}$ & 6.22 & 2.93 \\
Social & & & $0.53^{* *}$ & 10.02 & 4.53 \\
Psychological & & & & 12.41 & $5.5 \mathrm{I}$ \\
\hline
\end{tabular}

Note: $* * P<0.01$

Abbreviations: M, mean; SD, standard deviation.
Table 3 The results of multivariate analysis of covariance

\begin{tabular}{llllll}
\hline & Pillai's trace & $\boldsymbol{F}$ & $\mathbf{d f}$ & $\eta^{2}$ & Observed power \\
\hline Age group & 0.06 & $6.95 * * *$ & $3-317$ & 0.06 & 0.98
\end{tabular}

Notes: $* * * P<0.001$. Adjusted for marital status, sex, and employment status.

Abbreviations: $\mathrm{df}$, degrees of freedom; $\eta^{2}$, partial eta-squared.

adults and young people on the combined positive emotional health subscales $\left(F_{(3,317)}=6.95 ; P<0.001 ; \eta^{2}=0.06\right)$. The partial eta-squared $\left(\eta^{2}=0.06\right)$ was of medium size. No main effect was observed for marital status, sex, and employment status.

Finally, univariate analyses for each dependent variable as follow-up tests to the MANCOVA, using the Bonferroni method for controlling type I error rates, were conducted to examine individual mean difference across two groups and well-being subscales. As shown in Table 4, the results revealed a significant mean difference between older adults and young people in emotional well-being ( $\left.F=9.67 ; P<0.01 ; \eta^{2}=0.029\right)$, social well-being $(F=10.60$; $\left.P<0.001 ; \eta^{2}=0.032\right)$, and psychological well-being $\left(F=19.45 ; P<0.001 ; \eta^{2}=0.057\right)$.

On the basis of the estimated marginal means and the results of Sidak's $t$-test, older adults scored significantly higher on the emotional ( $\mathrm{M}=7.32$ vs $\mathrm{M}=6.00, P<0.01$ ), social $(\mathrm{M}=11.80$ vs $\mathrm{M}=9.65, P<0.01)$, and psychological $(\mathrm{M}=15.29$ vs $\mathrm{M}=11.81, P<0.001)$ well-being than young group, after adjustment for covariates.

Overall, MANCOVA results indicated significant group differences in positive emotional health, wherein elderly people scored higher on the positive emotional health scale than younger earthquake survivors.

\section{Discussion}

This study compared long-term psychological impact of earthquake experience between older adult group (aged 60-85 years) and young adult group (aged 18-30 years). After adjusting for marital status, sex, and employment status, the combined dependent variable of emotional, social,

Table 4 Results of univariate analyses for each dependent variable

\begin{tabular}{lllll}
\hline Dependent variable & $\mathbf{d f}$ & $\boldsymbol{F}$ & $\eta^{2}$ & $\begin{array}{l}\text { Observed } \\
\text { power }\end{array}$ \\
\hline Emotional well-being & $\mathrm{I}-319$ & $9.67^{* *}$ & 0.029 & 0.87 \\
Social well-being & $\mathrm{I}-319$ & $10.60^{* *}$ & 0.032 & 0.90 \\
Psychological well-being & $\mathrm{I}-319$ & $19.45^{* * *}$ & 0.057 & 0.99 \\
\hline
\end{tabular}

Notes: $* * p<0.01$; *** $p<0.001$.

Abbreviations: $d f$, degrees of freedom; $\eta^{2}$, partial eta-squared. 
and psychological well-being differed significantly between elderly group and young group. Older adults possessed a significantly higher adjusted mean emotional, social, and psychological well-being scores than young group.

Although some previous studies found that the older adults are more vulnerable to immediate impact of natural disasters and suffer more from injuries and loss of life in disasters than do younger people $\mathrm{e}^{2,23,24}$ and argued that the chronic health conditions, impaired cognitive abilities, and decreased sensory awareness that are correlated with age likely put older adults at a disadvantage in emergency situations, ${ }^{10,25}$ the results of the present study revealed that elderly survivors had higher levels of positive mental health compared to their younger counterparts.

The findings from the current study supported some studies that found the elderly survivors were less vulnerable to psychological problems. ${ }^{10,11}$ For example, the results from a study conducted 18 months after the 1988 earthquake in Armenia showed that no difference was observed on psychological disorders between the elderly and younger adults. Similarly, the results of an epidemiological study conducted after Hurricane Honduran revealed that the elderly survivors were at equal risk of developing psychological problems as the younger survivors. ${ }^{26}$

As mentioned in the Introduction section, the maturation theory and the inoculation theory argue that older adults are less vulnerable to disaster-related stress. The maturation theory proposes that in the face of disaster, older adults focus on more positive emotions than younger people do. The older adults are more resilient than the young adults, especially with respect to emotional regulation abilities and problem-solving abilities. ${ }^{27}$ The results from a study conducted to investigate psychological resilience in the older adults (aged $>64$ years) compared with their younger counterparts (aged $<26$ years) showed that the older adults were the more resilient group, especially with respect to emotional regulation ability and problem solving. ${ }^{27}$ The older people have a greater investment in emotional regulation. ${ }^{28}$ The inoculation theory suggests that the exposure to disaster earlier in life increases people's resistance to subsequent stress and reduces the impact of a disaster experienced late in life and may protect them from harm. From this perspective, elderly people would be predicted to experience less amount of post-disaster distress because they have experienced more stressful events than their younger counterparts. ${ }^{13,14,29}$

In summary, our study revealed that older adults, compared to younger adults, may be less vulnerable to longterm impacts of disasters. The present study is not free of limitations, which should be addressed and considered in future studies. 1) Due to cross-sectional nature of the study and the absence of comprehensive information about subjects, it is difficult to make a firm conclusion that elderly people are less psychologically vulnerable to natural disasters compared to younger age groups, because elderly people may have better mental health before disaster. ${ }^{30}$ 2) This survey used a two-stage proportional cluster random sampling technique resulting in unequal sample size groups that may affect results. Therefore, although variance was equal across groups and Box's M was not significant, the Pilli's criterion was used because it is more robust and appropriate when there are unequal sample sizes. In addition, the study sample size met the rule of thumb concerning unequal sample sizes, which argues that the variance ratio of the variance of the largest group to the variance of smallest group should be $3: 1$ or less. Another concern that should be acknowledged is that our survey entirely relies on the self-report of the individuals completing the questionnaires.

\section{Conclusion}

Contrary to some commonly held beliefs that aging is associated with increased dependency, loss of self-control, ailment, incapacity, disengagement from life, and social isolation that may prone them more vulnerable to life crises, the results of our study showed that elderly earthquake survivors have a higher positive mental health compared with their younger counterparts in the wake of natural disasters. According to the present study, it can be concluded that advancing age per se does not contribute to increasing vulnerability. Therefore, results of this study may have the following implications for those involved in providing disaster management. 1) Consider older people's knowledge and experience in developing coping strategies following disaster. 2) Involve older adults in the development of disaster prevention, preparedness, and management programs. 3) Elderly people can be considered as valuable resources in their families and communities to provide emotional support to other disaster victims.

\section{Acknowledgment}

The authors would like to thank all participants who generously contributed to the research. This study was financially supported by University of Social Welfare and Rehabilitation Sciences (grant number: 10245).

\section{Disclosure}

The authors report no conflicts of interest in this work. 


\section{References}

1. Blaikie P, Cannon T, Davis I, Wisner B. At Risk: Natural Hazards, People's Vulnerability and Disasters. London: Routledge; 2014.

2. Jia Z, Tian W, Liu W, Cao Y, Yan J, Shun Z. Are the elderly more vulnerable to psychological impact of natural disaster? A populationbased survey of adult survivors of the 2008 Sichuan earthquake. $B M C$ Public Health. 2010;10(1):1-11.

3. Lin MR, Huang W, Huang C, Hwang HF, Tsai LW, Chiu YN. The impact of the Chi-Chi earthquake on quality of life among elderly survivors in Taiwan-a before and after study. Qual Life Res. 2002; 11(4):379-388.

4. Alipour F, Khankeh H, Fekrazad H, Kamali M, Rafiey H, Ahmadi S. Social issues and post-disaster recovery: a qualitative study in an Iranian context. Int Soc Work. 2015;58(5):689-703.

5. Chan EY. The untold stories of the Sichuan earthquake. Lancet. 2008;372(9636):359-362.

6. Pekovic V, Seff L, Rothman MB. Planning for and responding to special needs of elders in natural disasters. Generations. 2007;31(4): $37-41$.

7. Alipour F, Khankeh HR, Fekrazad H, et al. Challenges for resuming normal life after earthquake: a qualitative study on rural areas of Iran. PLoS Curr. 2014;6:ecurrents.

8. Srinivasan S, Llorente MD, Magley M. Mental Health Consequences of Disaster Exposure in Older Adults. Disaster Preparedness for Seniors. New York, NY: Springer; 2014:311-327.

9. O'donnell ML, Forbes D. Natural disaster, older adults, and mental health-a dangerous combination. Int Psychogeriatr. 2016; 28(01):9-10.

10. Cherniack EP. The impact of natural disasters on the elderly. $A m$ J Disaster Med. 2008;3(3):133-139.

11. Goenjian AK, Najarian LM, Pynoos RS, et al. Posttraumatic stress disorder in elderly and younger adults after the 1988 earthquake in Armenia. Am J Psychiatry. 1994;151(6):895-901.

12. Banks L. Caring for elderly adults during disasters: improving health outcomes and recovery. South Med J. 2013;106(1):94-98.

13. Thompson MP, Norris FH, Hanacek B. Age differences in the psychological consequences of Hurricane Hugo. Psychol Aging. 1993;8(4):606-616.

14. Wang T, Yang H, Han B. A follow up study on the elderly survivors' mental health two years after the Wenchuan earthquake. World Acad Sci, Eng Technol, Int J Soc, Behav, Educ, Econ, Bus Ind Eng. 2012; 6(5):872-880.

15. Knight BG, Gatz M, Heller K, Bengtson VL. Age and emotional response to the Northridge earthquake: a longitudinal analysis. Psychol Aging. 2000;15(4):627-634
16. Lindert J, Levav I. Violence and Mental Health: Its Manifold Faces. New York, NY: Springer; 2015.

17. Seligman ME, Csikszentmihalyi M. Positive psychology: An introduction. The Netherlands: Springer; 2014:279-298.

18. Ardalan A, Babaie J, Moradian MJ, Shariati M, Yousefi H. Incorporating the lessons learned from the 2012 East Azerbaijan Earthquakes in Iran's National Health Emergency Plan. Prehosp Disaster Med. 2013;28(4): 417.

19. Joshanloo M, Wissing MP, Khumalo IP, Lamers SM. Measurement invariance of the Mental Health Continuum-Short Form (MHC-SF) across three cultural groups. Pers Individ Dif. 2013;55(7):755-759.

20. Guo C, Tomson G, Guo J, Li X, Keller C, Söderqvist F. Psychometric evaluation of the Mental Health Continuum-Short Form (MHC-SF) in Chinese adolescents-a methodological study. Health Qual Life Outcomes. 2015;13(1):198.

21. de Carvalho JS, Pereira NS, Pinto AM, Marôco J. Psychometric properties of the mental health continuum-short form: a study of Portuguese speaking children/youths. J Child Fam Stud. 2016;25(7):2141-2154.

22. Machado WdL, Bandeira DR. Positive Mental Health Scale: validation of the Mental Health Continuum-Short Form. Psico-USF. 2015;20(2):259-274.

23. Liu A, Tan H, Zhou J, et al. An epidemiologic study of posttraumatic stress disorder in flood victims in Hunan China. Can J Psychiatry. 2006;51(6):350-354.

24. Ticehurst S, Webster RA, Carr VJ, Lewin TJ. The psychosocial Impact of an earthquake on the elderly. Int $J$ Geriatr Psychiatry. 1999;11(11):943-951.

25. Lindell MK, Tierney KJ, Perry RW. Facing the Unexpected: Disaster Preparedness and Response in the United States. Washington, DC: Joseph Henry Press; 2001.

26. Kohn R, Levav I, Garcia ID, Machuca ME, Tamashiro R. Prevalence, risk factors and aging vulnerability for psychopathology following a natural disaster in a developing country. Int $J$ Geriatr Psychiatry. 2005;20(9):835-841.

27. Gooding P, Hurst A, Johnson J, Tarrier N. Psychological resilience in young and older adults. Int J Geriatr Psychiatry. 2012;27(3): $262-270$.

28. Kuh D, Network NDoAP. A life course approach to healthy aging, frailty, and capability. J Gerontol A Biol Sci Med Sci. 2007;62(7):717-721.

29. Norris FH, Murrell SA. Prior experience as a moderator of disaster impact on anxiety symptoms in older adults. Am J Community Psychol. 1988;16(5):665-683.

30. Momtaz YA, Hamid TA, Ibrahim R. Cohort comparisons: emotional well-being among adolescents and older adults. Clin Interv Aging. 2014;9:813-819.
Clinical Interventions in Aging

\section{Publish your work in this journal}

Clinical Interventions in Aging is an international, peer-reviewed journal focusing on evidence-based reports on the value or lack thereof of treatments intended to prevent or delay the onset of maladaptive correlates of aging in human beings. This journal is indexed on PubMed Central, MedLine,

\section{Dovepress}

CAS, Scopus and the Elsevier Bibliographic databases. The manuscript management system is completely online and includes a very quick and fair peer-review system, which is all easy to use. Visit http://www.dovepress. com/testimonials.php to read real quotes from published authors. 